\title{
EVALUACION CUALITATIVA DEL CONSERVADURISMO CONTABLE EN CHILE
}

\section{QUALITATIVE ASSESSMENT OF ACCOUNTING CONSERVATISM IN CHILE}

\author{
Valeria G. Perez Stizzoli ${ }^{1}$ y Germán R. Pinto Perry ${ }^{2}$
}

\section{RESUMEN}

El concepto de prudencia o conservadurismo ha sido estudiado en diversas investigaciones tanto desde la perspectiva teórica, como en su aplicación práctica en la revelación de la información contable: Basu [(1997), (2005)], Pope and Walker (2003), Watts (2003), Beaver and Ryan (2005), Brown et al (2006), García Lara and Mora (2004), entre otros.

En tanto, el IASB ha readecuado su Marco Conceptual en el año 2010, eliminando el concepto de prudencia, pese a que aún subsisten prácticas prudenciales que están reguladas en sus propias normas (conservadurismo normativo).

Esa preocupación por el tema también es apreciado en la revisión de literatura que hemos realizado, la cual evidencia en estudio de cómo las variables que las investigaciones empíricas realizadas, se constatan en la revelación en las notas de los estados contables de diversas entidades en distintos países, lo que nos ha motivado a realizar un estudio sobre la forma de materialización de los criterios conservadores que se aprecian en la emisión de informes de la realidad económica y financiera de las entidades en Chile.

Es por ello que se realiza una investigación del tipo cualitativo basada en la observación de la información revelada y relatos de cómo se expresan en las notas de los estados contables los antecedentes útiles para apreciar los efectos del conservadurismo condicional e incondicional.

Esta revisión se realizó en 10 sociedades anónimas abiertas clasificadas en el IPSA, las cuales fueron consideradas como informantes claves.

Es posible concluir que existen diversas actitudes o maneras de revelar el conservadurismo en Chile, lo que se evidencia en el énfasis que demuestran las empresas para publicitar la información en las notas sobre este hecho, como también en los formatos utilizados en las diversas memorias anuales de esas entidades.

Palabras claves: Conservadurismo, información contable, NIIF, patrimonio neto, investigación cualitativa.

Recepción: 18/12/2015. Aprobación: 12/03//2016.

\section{ABSTRACT}

The concept of prudence or conservatism has been studied in various investigations both from a theoretical perspective, and their practical application in the disclosure of accounting information: Basu [(1997), (2005)], Pope and Walker (2003), Watts (2003), Beaver and Ryan (2005), Brown et al (2006), Garcia Lara and Mora (2004), among others.

1 Facultad de Ciencias Económicas de la Universidad Nacional del Litoral (UNL), Santa Fe, Argentina, email: vperez@fce.unl.edu.ar

2 Departamento de Contabilidad y Auditoría, Facultad de Administración y Economía, Universidad de Santiago de Chile, e-mail: german.pinto@ usach.cl 
While, the IASB has modified its framework in 2010, eliminating the concept of prudence, despite still existing prudential practices are governed by their own rules (regulatory conservatism).

That concern over the issue is also appreciated in the literature review we have done, which evidenced by studying how variables empirical research, are apparent in the disclosure in the notes to the financial statements of different entities in different countries This has motivated us to conduct a study on how to materialize the conservative criteria that can be seen in the issuance of reports of economic and financial reality of the entities in Chile.

For this reason, this research is qualitative based on observation of revealed and stories of how they are expressed in the notes to the financial statements useful background for assessing the effects of the conditional and unconditional conservatism information is performed.

This review was conducted in 10 publicly traded companies classified in the IPSA, which were considered key informants.

We conclude that there are various ways to reveal attitudes or conservatism in Chile, which is evident in the emphasis that companies show to publicize the information in the notes to this fact, as in the formats used in the various annual reports those entities.

Keywords: conservatism, accouting information, IFRS, accouting equity, qualitative research.

\section{INTRODUCCIÓN}

En el año 2010 el IASB (International Accounting Standard Board) ha readecuado su Marco Conceptual en virtud de acuerdos con otros organismos internacionales, eliminando del mismo el concepto de prudencia (Gebhardt et al, 2014, p.111). El fundamento a estos cambios está en las investigaciones y observaciones de profesionales, investigadores y doctrinarios contables, que indicaban que el concepto de prudencia conllevaba a prácticas contables relacionadas con la contabilidad creativa, cuando se exageraba o manipulaban las causas para realizar provisiones (Mejía, 2011, p. 69).

El término de prudencia era relacionado con el denominado conservadurismo, el cual "tiende a entrar en conflicto con importantes características cualitativas, como la fidelidad de representación, la neutralidad y la comparabilidad (incluida la coherencia)" (Mejía, 2011, p.72), causando contradicción con la cualidad de neutralidad en la información contable (Silva, 2012).

Sin embargo, existe una errónea interpretación del término conservadurismo (Mora y Walker, 2015), pues, la excesiva utilización de prudencia en la información contable no es conservadurismo sino manipulación de la información. Asimismo, existen aspectos particulares de ésta que, por su naturaleza y su subordinación a determinadas regulaciones (regulación fiscal, medioambiental, de entidades financieras, etc), presentan prácticas conservadoras en la presentación de la información que emanan de las normas de los organismos controladores.
Por ello, el propósito de este trabajo es realizar una evaluación de las revelaciones en la información revelada, a través de la observación de las notas a los estados contables, evaluándose la forma de cómo se muestran (no del efecto que realmente generan en los tomadores de información) y la evaluación del fundamento de las prácticas contables conservadoras realizadas. De esta forma podemos apreciar el énfasis que demuestran las entidades al aplicar los imperativos normativos, lo que evidenciaría la mayor o menor preocupación por este ejercicio, que sería el pábulo del criterio conservador de los emisores de los estados contables.

Luego del análisis de la revisión de literatura que se efectuó para esta investigación, nació la interrogante si las variables determinadas por las investigaciones empíricas realmente determinaban la aplicación del conservadurismo en Chile o si existían otros criterios que pudieran hacer variar los resultados de las empresas en ese país a través del énfasis demostrado en la redacción y exposición de la información contenida en las notas a los estados contables. Es por ello que surge la motivación de evaluar cualitativamente este hecho, basado en la apreciación de la calidad de la información evaluando la manera de cómo se revela información útil vinculada al conservadurismo.

En este sentido se plantea la siguiente pregunta de investigación: ¿Existe alguna diversidad en la forma de la expresión de la información contable revelada en los estados contables que pudiera evidenciar una diferencia en la aplicación del conservadurismo en las empresas que 
cotizan en Chile?

Para ello, en esta investigación aplica el enfoque cualitativo, realizando una observación de los datos numéricos y la forma de relatar las notas de los estados contables para apreciar la aplicación de las normas de Contabilidad conservadoras, a fin de apreciar los efectos del conservadurismo condicional e incondicional en la información contable.

Se optó por concentrar la observación en la información entregada en las notas a los estados contables, porque son éstas las que gozan de una libertad para su redacción y elaboración, permitiendo, a través de esta libertad, la exposición del mayor o menor grado de conservadurismo en las entidades analizadas.

\section{REVISIÓN DE LITERATURA}

El concepto de prudencia o conservadurismo ha sido analizado en diversas investigaciones contables empíricas, tanto desde la perspectiva teórica, así como sus implicancias en las revelaciones de la información contable: Basu [(1997), (2005)], Pope and Walker (2003), Watts (2003), Beaver and Ryan (2005), Brown et al (2006), García Lara and Mora (2004), por mencionar solo algunas investigaciones sobre la temática.

El marco conceptual de la IASB (International Accounting Standard Board) consideraba el concepto de prudencia entre las cualidades de la información económica y financiera para la presentación de los estados contables. En este sentido, se pronunciaba como:

"la inclusión de un cierto grado de precaución en el ejercicio de juicios necesarios para efectuar las estimaciones requeridas bajo condiciones de incertidumbre, de modo que los activos o los ingresos no se expresen en exceso y que las obligaciones y los gastos no se expresen en defecto" (Mejías, 2011, p. 71).

En el año 2010, el IASB readecuó su Marco Conceptual en virtud de acuerdos con otros organismos internacionales (Gebhardt et al, 2014), eliminando el concepto de prudencia, a fin de no generar contradicción con la cualidad de neutralidad de la información contable (Silva, 2012), y evitar la errónea interpretación del término y las consecuencias por su implementación en exceso, llevando incluso a situaciones de manipulación de la información contable (García Lara et al, 2005).

Sin embargo, el concepto de prudencia aún se encuentra en otros marcos conceptuales en la actualidad, como es el caso de Argentina, en cuya normativa profesional RT $\mathrm{N}^{\circ} 16$ FACP$\mathrm{CE}$ define en su punto 3.1.2.1., Aproximación a la Realidad, como atributo de la información contable, en el que incorpora una aclaración al término "prudencia" y su diferenciación del término conservadurismo.

De las investigaciones sobre conservadurismo se observan dos conceptos importantes: conservadurismo incondicional y conservadurismo condicional.

El conservadurismo incondicional, de balance, ex ante, o también denominado ajeno a las noticias, es aquel que "en el que los procesos contables no reconocen ciertos aspectos de rendimiento esperado en la incorporación de activos y pasivos" ${ }^{3 "}$ (Beaver, W.H. \& Ryan, S.G., 2005 , p. 269), estableciendo políticas conservadoras en el tiempo. Específicamente, el concepto de conservadurismo de balance podría entenderse como la subvaluación del patrimonio neto con respecto al valor de mercado de la empresa (Íñiguez et al, 2013, p. 454), sea porque no se reconocen ciertos activos (como pueden ser ciertos activos intangibles, investigación y desarrollo), o por la infravaloración de activos o sobrevaloración de pasivos (contingencias negativas, políticas de gestión de riesgos).

Por otra parte, el conservadurismo condicional, de resultado, ex post, o de noticias (condicionado a las noticias), puede ser definido como "el valor contable -de libro- (que) reconoce las circunstancias adversas - malas noticias - antes que las favorables - buenas noticias $-{ }^{\prime \prime 4}$ (Beaver, W.H. \& Ryan, S.G., 2005, p. 269), la decisión de valorización incide en el resultado de la información contable (como pueden ser los deterioros en el modelo de costo o deterioro del fondo de comercio). Este tipo de conservadurismo ha sido el más analizado entre las investigaciones empíricas, destacándose la aplicación del modelo de Basu (1997) entre los mayores aportes para su análisis. En esta investigación, Basu evidenció que las malas noticias son reconocidas más oportunamente que las buenas noticias en los resultados de la información contable, ya que el mercado reacciona tanto a las noticias buenas como a las malas. 
Asimismo, diversos estudios, desde la perspectiva empírica, han analizado los determinantes del conservadurismo contable. Así trabajos como los de Watts (2003), Basu (2005), Ball (2008), evidenciaron que los factores que influyen en el nivel de conservadurismo son: factores institucionales, estructura normativa del sistema contable, los contratos de deuda, la influencia fiscal (regulación fiscal), el riesgo de litigio (de gerencia, de situaciones de riesgo, de auditores), el escrutinio regulador (organismos reguladores prudenciales).

Ambos conceptos de conservadurismo tienen componentes no discrecionales (según las normativas contables) y discrecionales (estimaciones de la gerencia ante situaciones de incertidumbre) (Mora y Walker, 2015).

Se entiende por componente no discrecional a la aplicación del conservadurismo según expresamente lo señalan las normas contables.

Se entiende por componente discrecional a la estimación en la aplicación de las prácticas conservadoras contempladas en las normas contables, es decir, cuando la administración discierne sobre cómo aplicar el imperativo normativo conservador.

\subsection{Conservadurismo Normativo}

Se entiende por conservadurismo normativo a las prácticas contables conservadoras que se realizan según lo establecen las normas contables, tal como serían los casos que se detallan a continuación:

La NIC 36 regula el reconocimiento del deterioro del valor de los activos. Este deterioro "surge cuando el valor libros de un activo es mayor que el valor asociado a su servicio recuperable" (Ruz Farías, 2008, p. 497). En esta situación, la normativa obliga al ente a efectuar una estimación formal del cálculo del valor recuperable del activo.

La NIC 37, considera las situaciones de provisiones, contingencias (de activos y pasivos), en sus párrafos 84 a 92. Esta norma requiere que el ente revele movimientos, montos, descripción de la naturaleza de la situación contingente, y, de no ser posible, revelar esa información como excepcional. En este último caso, solo se deberá informar una descripción genérica de la naturaleza de la situación, detallando las razones por las que no se ha revelado en forma completa.

En la NIIF 9 se puede observar el actual modelo de reconocimiento de deterioro crediticio, con el que se pueden realizar las estimaciones sobre las pérdidas futuras de los créditos, reco- nociendo el deterioro de los mismos según la existencia de evidencia objetiva. Así, si esas estimaciones fueran a producir un exceso de provisiones, dando la infravaloración deliberada en el activo, no sería conservadurismo sino manipulación de la información contable.

Cabe aclarar que la aplicación de la NIIF 9 ha sido prorrogada a partir de los ejercicios iniciados el 1 de enero de 2018, por lo que aún está vigente la aplicación de la NIC 39, y, en relación a las ganancias o deterioros de los activos financieros durante un periodo económico, la empresa debe revelar en la información financiera o notas explicativas, las que fueran reconocidas, según lo especifica el párrafo 20 de la NIIF 7.

A su vez, la NIIF 7, en su párrafo 36, determina si se utilizará una cuenta correctora para reducir el valor en libros de los activos causados por deterioros, debiendo revelarse los criterios adoptados para ello (Ruz Farías, 2008, p. 264).

\section{OBJETIVO}

Evaluar cómo revelan en los informes contables las prácticas contables conservadoras las empresas chilenas bajo IFRS.

\section{METODOLOGÍA}

La investigación se desarrolla bajo el paradigma cualitativo, en la que se requiere una "labor básicamente hermenéutica, en procedimientos básicos como la descripción en profundidad y la exploración temática, ambas con un esfuerzo interpretativo y comprensivo, según las variantes internas que se han legitimado en este paradigma" (Patiño y Rojas, 2011, p. 20).

El objeto de la metodología cualitativa es el conocimiento de la realidad, accediendo a ella a través del discurso. Por discurso se entiende todo texto producido por alguien en una situación de comunicación interpersonal, sea esta oral, escrita o filmada (Báez y Pérez de Tudela, 2007; p37).

Se ha elegido este paradigma cualitativo porque se ha apreciado que el tema del conservadurismo contable ha sido principalmente abordado desde una dimensión cuantitativa, la cual se circunscribe al supuesto de la correlación entre determinadas variables. Sin desmerecer este hecho, nuestra propuesta surge para aportar elementos que permitan incluir nuevas variables en los modelos de análisis que campean en el mundo académico, como también para evaluar 
su efectividad según la realidad analizada.

Basu (1997) establece una relación entre la rentabilidad de las acciones y la aplicación de criterios conservadores, asumiendo que la información contable reconoce en el resultado contable primeramente las malas noticias que las buenas. Sin embargo, las características de la información contable que revelan los agentes económicos son diversas, dado el componente discrecional del conservadurismo que hemos señalado anteriormente, pudiendo apreciarse una diversidad en las formas cómo se revelan las aplicaciones de deterioro, provisiones y estimaciones. De esta forma, se espera ilustrar la diversidad en las formas cómo revelar las provisiones y demás ajustes que las normas obligan a realizar, evidenciando que la discreción con la cual se realiza, redunda en una multiplicidad de estilos que pueden afectar de diversas formas las decisiones de inversión que están basadas o influenciadas por el conservadurismo contable.

Es importante señalar que el deterioro está vinculado a la coyuntura económica particular en el tiempo y podría suponerse que hay más o menos conservadurismo (evidenciado con la aplicación del deterioro) según esta variable. Este hecho, que pudiera ser aceptado lógicamente, no es un elemento a considerar en esta investigación porque la coyuntura económica es una variable transversal a todas las entidades en un momento dado, siendo un verdadero punto de partida para que ellas reaccionen y revelen. Ese ejercicio de revelación se realiza según distintos criterios y estilos que permiten diferenciarlos y, por ende, evaluar el distinto criterio en la aplicación del conservadurismo. Es por ello que la coyuntura económica no es una variable que consideremos como factor que genera efectos en la evaluación que se realizará.

Por todas las consideraciones anteriores, se ha desarrollado la metodología consistente en evaluar a través de la observación y descripción de la información revelada en notas a los estados contables de 10 sociedades anónimas abiertas clasificadas en el IPSA, considerándolas como informantes claves. Su selección se basó en una estimación de representatividad de las 40 empresas que forman el IPSA, estableciendo la siguiente distribución:

Tabla $N^{\circ} 1$. Elaboración Propia.

\begin{tabular}{|l|c|c|c|}
\hline \multicolumn{1}{|c|}{ Tipo de Empresas } & Num.Empresas & Porcentaje & Num.Muestra \\
\hline Sociedades financieras & 6 & $15 \%$ & 2 \\
\hline Sociedades comerciales & 21 & $53 \%$ & 4 \\
\hline Sociedades de inversiones & 8 & $20 \%$ & 2 \\
\hline Sociedades de retail & 2 & $2 \%$ & 1 \\
\hline Otras & 3 & $10 \%$ & 1 \\
\hline Total & 40 & $100 \%$ & 10 \\
\hline
\end{tabular}

Esta tabla evidencia el criterio para seleccionar las empresas que fueron consideradas como informantes claves, las que se seleccionaron según la clasificación por tipo de empresas que se utilizó como criterio de segregación. La elección de cada una obedeció a un criterio intencional, valorando el tamaño y la calidad de la información que revelaban en sus notas a los estados contables. Esta selección intencional no es un desmedro a la objetividad de la investigación, pues, dado su carácter cualitativo, la atención está en los atributos de los elementos seleccionados.

Obviamente, esta selección circunscribe los resultados obtenidos a este grupo de 10 perso- nas, siendo posible extrapolar sus criterios según los atributos de acuerdo a los criterios propios de la investigación cualitativa (Fernández y Díaz, 2002). Cabe destacar que en el paradigma cualitativo "la cantidad es parte de la cualidad, además de darse mayor atención a lo profundo de los resultados y no a su generalización" (Urreola, 2011).

Las herramientas que se utilizaron son la observación y la descripción, poniendo atención en cómo se revelaban en las notas los imperativos generales de las NIC con algún sesgo del conservadurismo. Además, se hizo un análisis determinando la frecuencia de aparición de la palabra "deterioro" en la redacción de las notas 
de los estados contables, asumiendo que este vocablo representa una acción positiva (evidente, empírica) de que se está aplicando el criterio conservador.

La información utilizada fue recogida de las memorias publicadas para el ejercicio comercial 2014.

Asimismo, este trabajo está focalizado en el estudio de los componentes discrecional y no discrecional en el conservadurismo, analizando las normas aplicadas (componente no discrecional) en las entidades que cotizan en bolsa, y evaluando cómo revelan en las notas de la información contable (componente discrecional), situaciones que obedecen a esta naturaleza.

Si bien el título de la investigación señala que es la evaluación del conservadurismo en Chile, se hace una investigación cualitativa (insistimos en este carácter) sobre la base de la observación de las empresas analizadas que, se cree, que es suficiente para su extrapolación.

\section{RESULTADOS}

De la observación y descripción de la información revelada en notas a los estados contables de las 10 sociedades anónimas abiertas clasificadas en el IPSA que se tomaron de muestra, se presentan:

En el Cuadro I, los resultados del análisis de la frecuencia de la palabra "deterioro" y para el estudio de los componentes discrecional (cómo revelan en las notas de la información contable) y no discrecional (analizando las normas aplicadas) en el conservadurismo, se consideran los siguientes puntajes:

- 0 : el atributo no se constata en la nota.

- 1: el atributo se constata, obedece a una revelación a través de un texto, el cual, según hemos convenido, no genera un impacto mayor en el lector de los estados contables, que el que produce una revelación numérica.

- 2: el atributo se constata, y obedece a una revelación a través de un cuadro o referencia numérica, los cuales hemos convenido produce un impacto mayor en el lector de los estados contables.

Dado los parámetros anteriores, se obtendrá que la empresa que tenga un mayor puntaje sea aquella que revela en mayor forma hechos cuantitativos vinculados al conservadurismo.

En virtud que la norma contable solo señala la obligación de hacer una revelación sin precisar el formato, y menos señalar si esta revelación es a través de un texto o de un cuadro numérico, se considera que el elemento discrecional está presente con mayor énfasis en aquellas revelaciones a través de cuadros que señalen expresamente el efecto en resultados. Es por ello, que se otorga puntaje 2 (máximo) al que constata este atributo. 
Cuadro I: Análisis de la revelación de conservadurismo en notas a los estados contables y frecuencia de la palabra "deterioro".

\begin{tabular}{|c|c|c|c|c|c|c|c|c|c|c|}
\hline Atributo & $\begin{array}{l}5 \\
3 \\
3 \\
3\end{array}$ & 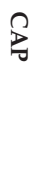 & 占 & $\begin{array}{l}\text { 刍 } \\
\text { 党 }\end{array}$ & 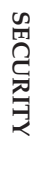 & $\hat{\imath}$ & $\begin{array}{l}\text { 罗 } \\
\text { 罗 } \\
\text { D }\end{array}$ & 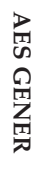 & 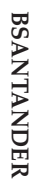 & 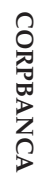 \\
\hline $\begin{array}{l}\text { Tiene nota de "gestión de riesgo" que señala criterios en forma } \\
\text { de texto }\end{array}$ & 1 & 1 & 1 & 1 & 1 & 1 & 1 & 1 & 1 & 1 \\
\hline $\begin{array}{l}\text { Tiene nota de "gestión de riesgo" que señala cuadros que evi- } \\
\text { dencian efecto en resultados }\end{array}$ & 0 & 0 & 0 & 0 & 0 & 0 & 0 & 0 & 0 & 0 \\
\hline $\begin{array}{l}\text { Tiene nota que evidencie estimaciones y juicios contables con } \\
\text { revelaciones numéricas }\end{array}$ & 0 & 0 & 0 & 2 & 0 & 0 & 0 & 0 & 2 & 2 \\
\hline $\begin{array}{l}\text { Tiene nota que evidencie estimaciones y juicios contables con } \\
\text { revelaciones en textos }\end{array}$ & 1 & 1 & 0 & 0 & 0 & 1 & 0 & 0 & 0 & 0 \\
\hline $\begin{array}{l}\text { Tiene nota de "deudores comerciales" que señala numérica- } \\
\text { mente el deterioro y provisiones por incobrabilidad }\end{array}$ & 2 & 2 & 0 & 2 & 0 & 0 & 2 & 0 & 0 & 0 \\
\hline $\begin{array}{l}\text { Tiene nota de deudores comerciales que evidencie castigos lle- } \\
\text { vados a resultados, destacando su efecto en resultados }\end{array}$ & 2 & 2 & 0 & 0 & 2 & 0 & 0 & 0 & 2 & 2 \\
\hline $\begin{array}{l}\text { Tiene nota de "Propiedades, planta y equipos" que señale el } \\
\text { cargo a resultados por depreciación }\end{array}$ & 2 & 2 & 2 & 2 & 2 & 0 & 0 & 0 & 0 & 2 \\
\hline $\begin{array}{l}\text { Tiene nota de "Propiedades, planta y equipos" que detalle el } \\
\text { deterioro }\end{array}$ & 0 & 0 & 0 & 0 & 2 & 0 & 2 & 0 & 0 & 2 \\
\hline $\begin{array}{l}\text { Tiene nota de "Propiedades, planta y equipos" que señale el } \\
\text { deterioro a través de cifras numéricas }\end{array}$ & 0 & 2 & 2 & 0 & 0 & 0 & 0 & 0 & 2 & 2 \\
\hline $\begin{array}{l}\text { Tiene nota de "Instrumentos Financieros" en donde revele de- } \\
\text { terioro en forma numérica }\end{array}$ & 0 & 2 & 2 & 2 & 2 & 0 & 0 & 2 & 2 & 2 \\
\hline $\begin{array}{l}\text { Tiene nota de "Instrumentos Financieros" en donde señale cri- } \\
\text { terios de deterioros o ajustes en forma de texto }\end{array}$ & 0 & 1 & 0 & 0 & 1 & 1 & 1 & 0 & 0 & 0 \\
\hline $\begin{array}{l}\text { Tiene nota para "activos intangibles" que señalen algún efecto } \\
\text { en resultados revelado en forma numérica }\end{array}$ & 0 & 2 & 2 & 0 & 2 & 0 & 0 & 0 & 0 & 2 \\
\hline $\begin{array}{l}\text { Tiene nota para "activos intangibles" que señale algún criterio } \\
\text { conservador a través de un texto }\end{array}$ & 0 & 1 & 0 & 0 & 0 & 1 & 0 & 0 & 0 & 0 \\
\hline $\begin{array}{l}\text { Tiene una nota sobre "activos y pasivos de cobertura" que seña- } \\
\text { la el efecto en resultados en forma numérica }\end{array}$ & 0 & 0 & 2 & 0 & 0 & 0 & 0 & 0 & 0 & 2 \\
\hline $\begin{array}{l}\text { Tiene una nota sobre "activos y pasivos de cobertura" que seña- } \\
\text { la el efecto en resultados en forma de texto }\end{array}$ & 0 & 0 & 0 & 0 & 0 & 0 & 0 & 0 & 0 & 0 \\
\hline $\begin{array}{l}\text { Tiene alguna nota que evidencie algún efecto en resultados por } \\
\text { medición a valor razonable a través de un cuadro numérico }\end{array}$ & 0 & 0 & 0 & 0 & 0 & 0 & 0 & 0 & 0 & 0 \\
\hline $\begin{array}{l}\text { Tiene alguna nota que evidencie algún efecto en resultados por } \\
\text { medición a valor razonable a través de un texto }\end{array}$ & 0 & 0 & 1 & 0 & 0 & 0 & 0 & 0 & 0 & 0 \\
\hline $\begin{array}{l}\text { Tiene una nota particular que detalla cada partida que forma } \\
\text { parte del estado de resultados por función de gastos y que evi- } \\
\text { dencia el deterioro en algunas partidas }\end{array}$ & 0 & 0 & 2 & 0 & 0 & 0 & 0 & 2 & 0 & 0 \\
\hline $\begin{array}{l}\text { Tiene una nota particular que detalla los arriendos entre em- } \\
\text { presas relacionadas, destacando que se trata de una utilidad no } \\
\text { realizada. Esto se detalla numéricamente }\end{array}$ & 0 & 0 & 0 & 2 & 0 & 0 & 0 & 0 & 0 & 0 \\
\hline $\begin{array}{l}\text { Tiene una nota especial de inventarios donde detalla numérica- } \\
\text { mente el valor neto, pero no detalla efecto en resultados }\end{array}$ & 0 & 0 & 0 & 1 & 0 & 0 & 1 & 0 & 0 & 0 \\
\hline $\begin{array}{l}\text { Tiene una nota especial para inventarios donde se detalla infor- } \\
\text { mación a través de texto sin evidenciar efecto en resultados }\end{array}$ & 0 & 0 & 0 & 0 & 0 & 0 & 0 & 1 & 0 & 0 \\
\hline $\begin{array}{l}\text { Tiene una nota especial para revelar el deterioro aplicado a todas } \\
\text { las partidas del balance }\end{array}$ & 0 & 0 & 0 & 0 & 2 & 0 & 2 & 0 & 0 & 0 \\
\hline Frecuencia de palabra "deterioro" & 28 & 72 & 57 & 43 & 92 & 55 & 52 & 40 & 51 & 160 \\
\hline TOTAL & 36 & 88 & 71 & 55 & 106 & 59 & 61 & 46 & 60 & 177 \\
\hline
\end{tabular}

Fuente: Elaboración propia. 
El Cuadro II expone la conclusión general a la que se arriba luego de realizar la observación empírica (extraída de la realidad) de las notas de los estados contables expuestos respecto de las revelaciones de las prácticas contables conservadoras. Se presenta este cuadro como una especie de resumen de la realidad observada en cada caso.

Cuadro II: Comentario general según análisis realizado sobre la revelación de conservadurismo en notas a los estados contables.

\begin{tabular}{|c|c|}
\hline Empresa & Comentario General \\
\hline LATAM & $\begin{array}{l}\text { Esta empresa cumple con el mínimo exigido por la norma y no evidencia una pre- } \\
\text { ocupación especial para revelar aspectos conservadores en sus estados contables }\end{array}$ \\
\hline CAP & $\begin{array}{l}\text { Dada la forma cómo se revelan y cómo se muestran tablas que ilustran el deterioro } \\
\text { de algunas partidas, se puede concluir que esta empresa dedica una importante } \\
\text { atención a revelar rubros como activos intangibles y propiedades, plantas y equi- } \\
\text { pos que son sensibles al deterioro, provisiones y depreciación. }\end{array}$ \\
\hline SQM-B & $\begin{array}{l}\text { SQM-B no es una empresa que evidencie en sus notas un conservadurismo más } \\
\text { destacado que el mínimo exigido por las normas contables. Es más, revela un mí- } \\
\text { nimo suficiente }\end{array}$ \\
\hline RIPLEY & $\begin{array}{l}\text { Se evidencia que Ripley cumple con el mínimo exigido para evidenciar el efecto en } \\
\text { sus resultados por la aplicación de criterios conservadores, exponiendo lo mínimo } \\
\text { necesario sin aportar alguna información de mayor calidad para que los usuarios } \\
\text { puedan apreciar alguna situación negativa para la empresa. }\end{array}$ \\
\hline SECURITY & $\begin{array}{l}\text { Se aprecia que esta compañía cumple el mínimo exigido por las normas, no siendo } \\
\text { destacable algo más allá de lo normado }\end{array}$ \\
\hline $\mathrm{CCU}$ & $\begin{array}{l}\text { Esta empresa cumple con el mínimo exigido por la norma y no evidencia una pre- } \\
\text { ocupación especial para revelar aspectos conservadores en sus estados contables. }\end{array}$ \\
\hline ENDESA & $\begin{array}{l}\text { La revelación de aspectos conservadores se vislumbra en el rubro provisiones en } \\
\text { virtud de las exigencias de la regulación medioambiental y relacionada a la ac- } \\
\text { tividad principal de esta entidad. Por el resto de las revelaciones no demuestra } \\
\text { mayores detalles de situaciones conservadoras. }\end{array}$ \\
\hline AES GENER & $\begin{array}{l}\text { Esta entidad presenta en la revelación de la información financiera casi una nula } \\
\text { aplicación de situaciones conservadoras, salvo en el caso de provisiones donde se } \\
\text { revelan las situaciones que están regidas por algún contrato, regulación o leyes }\end{array}$ \\
\hline BSANTANDER & $\begin{array}{l}\text { Las revelaciones de las notas de estos balances presentan cuestiones de forma res- } \\
\text { pecto a las prácticas conservadoras, no haciendo mayores detalles y ajustándose } \\
\text { solo a algunos rubros a los que puedan aplicarse }\end{array}$ \\
\hline CORPBANCA & $\begin{array}{l}\text { Se destaca el nivel de detalle en la descripción, criterios, y ajustes por deterioro, en } \\
\text { los cuadros presentados y en el desarrollo de las notas en los estados financieros, } \\
\text { revelando las prácticas contables conservadoras. }\end{array}$ \\
\hline
\end{tabular}

Fuente: Elaboración propia.

\section{CONCLUSIONES}

Luego del análisis realizado, se concluye lo siguiente:

Existe un conservadurismo normativo que es aquel que se cumple por el solo hecho de res- petar las disposiciones establecidas en las NIIF, que representa el mínimo exigido a las entidades. Sin embargo, este cumplimiento no está expresamente normado ni regulado, pudiendo las emisoras aplicarlas según sus propios criterios y motivaciones. Esta brecha entre lo normado y lo revelado, es lo que puede ser evaluado cualita- 
tivamente para determinar qué entidad tiene un carácter más conservador que otras.

La revisión de literatura evidencia cuatro conceptos importantes:

- Conservadurismo condicional, aquel que afecta los resultados de la entidad, evidenciando en el resultado del ejercicio las decisiones conservadoras.

- Conservadurismo incondicional, que afecta las cuentas de balance y que altera el rendimiento de las partidas.

- Componente discrecional, aquel cumplimiento de lo establecido en las normas, según el criterio particular de cada administración, aplicable a la elección del método de valuación, de estimación o en la definición del tiempo cuando se reconocerán las pérdidas o ganancias.

- Componente no discrecional, aquel cumplimiento que está específicamente señalado en las normas.

El componente discrecional permite evaluar el grado del conservadurismo del emisor de una forma cualitativa, pues evidencia una diversidad de maneras, de cómo se ha cumplido el imperativo normativo. De esta forma, es posible apreciar que, de ser cierto que el conservadurismo puede ser medido según la rentabilidad de las acciones (del precio de los títulos que define el mercado), y, que esta ponderación se basa en la información que se entrega a los tomadores de decisiones, puede existir un factor inherente a esa información contable la cual puede estar expuesta de una forma tal que influya mayormente en ellos y, por ende, en la medición del conservadurismo según la concepción clásica de la forma de medir que se ha concebido.

Es posible concluir que existen diversas actitudes o maneras de revelar el conservadurismo en las empresas chilenas seleccionadas para la muestra, lo que se evidencia en el énfasis que demuestran los entes para revelar la información en las notas. Este se puede apreciar gracias a la diversidad de formatos y mínimos de datos a señalar en las notas a los estados contables. Esta libertad se evidencia en este énfasis.

Según la revisión realizada, se puede concluir que CORPBANCA es una empresa que demuestra más preocupación por revelar hechos que tienen efectos en resultados debido al deterioro, provisiones, estimaciones y otras consideraciones propias del conservadurismo. Según este análisis, sin considerar la frecuencia de la utilización de la palabra "deterioro", es la empresa que más información numérica y que evidencia efectos en resultados en mayor manera que las otras. Esta categoría sobresale incluso a otra empresa como BSANTANDER, compañía de la misma industria y que pudiera hacer pensar que las normas de la Superintendencia de Bancos e Instituciones Financieras (SBIF) sean más conservadoras que las normales emitidas por la Superintendencia de Valores y Seguros (SVS). Esto es tal, que incluso de ser así (que las normas de la SBIF sean más conservadoras que las de SVS), BSANTANDER no secunda a CORPBANCA en esta medición ya que en segundo lugar está SECURITY. Esta diversidad evidencia que existe distinta intensidad en la forma cómo se revela el conservadurismo las empresas chilenas observadas, siendo esto un antecedente importante a considerar en los estudios empíricos que puedan aplicarse la temática de conservadurismo.

Dado que existe una diversidad de formas para cómo revelar el conservadurismo en las empresas chilenas observadas, es posible afirmar que existe una distorsión en la apreciación directa de que el precio de la acción (o su rentabilidad) sea la conditio sine qua non para poder medir el conservadurismo, pues al haber una diversidad en la forma del "cómo" aplicar este conservadurismo, se puede colegir que existe un factor que haría alejar a los tomadores de decisiones de la directa correlación entre precio y valoración del conservadurismo.

\section{BIBLIOGRAFÍA}

Báez y Pérez de Tudela, J. (2007). Investigación cualitativa. Madrid: Esic Editorial.

Ball, R., Robin, A., Sadka, G., 2008. Is financial reporting shaped by equity markets or by debt markets? An international study of timeliness and conservatism. Review of Accounting Studies, $13(2-3), 168-205$.

Basu, S. (1997) The conservatism principle and the asymmetric timeliness of earning. Journal of Accounting and Economics, 24: 3-37.

Basu, S. (2005) Discussion of "conditional and unconditional conservatism: concepts and modelling" Review of Accounting Studies, 10 (2/3), 311-321 
Beaver, W.H. and Ryan, S.G. 2005. Conditional and Unconditional Conservatism: Concepts and Modelling. Review of Accounting Studies, 10 (2/3), 269-309.

Brown, W.D., Haigong, H., and Teitel, K. (2006) Conditional Conservatism and the value relevance of accounting earning: an international study. European Accounting Review, 15 (4), 605626.

Cabo-Moreno, I., Bautista-Mesa, R., MolinaSánchez, H., Ramírez-Sobrino, J. El papel de la contabilidad ante la crisis financiera. Una reflexión sobre el conservadurismo incondicional de la NIIF 9. Cuadernos de Contabilidad, 15 (38): 371-395, julio-diciembre 2014. Bogotá, Colombia.

Federación Argentina de Consejos Profesionales de Ciencias Económicas (FACPCE) (2012) Resolución Técnica $N^{\circ} 16$. Argentina: Errepar.

García Lara, J., García Osma, B. and Mora, A. 2005. The effect of earnings management on the asymmetric timely of earnings. Journal of Business Finance and Accounting, 32 (3/4), 691-726.

Garcia Lara, J.M., and Mora, A. (2004) Balance sheet versus earning conservatism in Europe. European Accounting Review, 13 (2), 261-292.

Gebhart, G., Mora, A., and Wagenhofer, A. (2014) Revisiting the Fundamental Concepts of IFRS. Abacus, 50 (1), 107-116.

Iñiguez, R., Poveda, F., Vázquez, P. (2013) Evolución del conservadurismo de balance con la implantación de las Normas Internacionales de Información Financiera: aplicación al caso de España. Revista Española de Financiación y Contabilidad. XLII, N 60, octubre-diciembre 2013, pp.453-486.

Mejías, J.F. (2011) La crisis de la prudencia en la convergencia contable internacional: Una mirada desde los deterioros de valor y las provisiones. Textos de Contabilidad Publica $\mathrm{N}^{\circ}$ 8, 67-95. Colombia.

Mora, A., Walker, M. (2015) The implacations of Research on Accounting Conservatism for Accounting Standards Setting. Conferencia en II Workshop Iberoamericano de Investigación Empírica en Contabilidad Financiera. Abril 2015, Universidad Nacional de Rosario, Argentina.
Patiño, L., Rojas, M. (2005) Métodos y enfoques en la investigación cualitativa. Colombia: Ed. El Poira Editores S.A.

Pita Fernández, S., \& Pértegas Díaz, S. (2002). Investigación cuantitativa y cualitativa. Cad Aten Primaria, 9, 76-8. Accedido el 17 de agosto de 2015 en http:/ / www.fisterra.com/mbe/investiga/cuanti_cuali/cuanti_cuali.asp

Pope, P. and Walker, M. (2003). Ex-ante and expost accounting conservatism, asset recognition and asymmetric earnings timeliness. Working Paper. Lancaster University and University of Manchester UK.

Ruz Farías, V. (2008). Una doctrina sobre las IFRS. Chile: RR Donnelley.

Silva, B. (2012) La información financiera y la importancia de su revelación. Octubre 2012. Accedido el 31 de mayo de 2015 en http:/ / www. researchgate.net/publication/259471185_LA_ INFORMACIN_FINANCIERA_Y_LA_IMPORTANCIA_DE_SU_REVELACIN

Urreola, R (2011) Integración y / o Confrontación de las Perspectivas Cualitativas y Cuantitativas en Investigación. Revista Razones Académicas. Publicación Digital de la Universidad ICEL, República del Ecuador. Año I Número 2 enero. Accedido el 11 de agosto de 2014 en http://www. icel.edu. $\mathrm{mx} / \mathrm{pdf} /$ razonesacademicas / razonesacademicas $02 /$ files/razones $\% 20$ academicas $\% 20$ $\underline{2 a}(1) \cdot p d f \#$ page $=11$

Watts, R.L. (2003) Conservatism in accounting Part I: Explanations and implications. Accounting Horizons, 17 (3), 207-221. 